\title{
Structure of the triose-phosphate/phosphate translocator reveals the basis of substrate specificity
}

Yongchan Lee ${ }^{1}$, Tomohiro Nishizawa ${ }^{1}$, Mizuki Takemoto ${ }^{1}$, Kaoru Kumazaki $^{1}$, Keitaro Yamashita ${ }^{2}$, Kunio Hirata ${ }^{2}$, Ayumi Minoda ${ }^{3}$, Satoru Nagatoishi ${ }^{4}$, Kouhei Tsumoto ${ }^{4}$, Ryuichiro Ishitani ${ }^{1}$, Osamu Nureki $^{1 *}$

Affiliations:

${ }^{1}$ Department of Biological Sciences, Graduate School of Science, The University of Tokyo, 2-11-16 Yayoi, Bunkyo-ku, Tokyo 113-0032, Japan.

${ }^{2}$ RIKEN SPring-8 Center, Hyogo 679-5148, Japan.

${ }^{3}$ Faculty of Life and Environmental Sciences, University of Tsukuba, 1-1-1 Tennodai, Tsukuba, Ibaraki 305-8572, Japan.

${ }^{4}$ Department of Bioengineering, School of Engineering, The University of Tokyo, 7-3-1 Hongo, Bunkyo-ku, Tokyo 113-8656, Japan.

*Correspondence: nureki@bs.s.u-tokyo.ac.jp 


\section{Abstract}

The triose-phosphate/phosphate translocator (TPT) catalyzes the strict 1:1 exchange of triose phosphate, 3-phosphoglycerate and inorganic phosphate across the chloroplast envelope, and plays crucial roles in photosynthesis. Despite rigorous studies for more than 40 years, the molecular mechanism of TPT is poorly understood due to the lack of structural information. Here we report crystal structures of TPT bound to two different substrates, 3-phosphoglycerate and inorganic phosphate, in occluded conformations. The structures reveal that TPT adopts a 10-transmembrane drug/metabolite transporter fold. Both substrates are bound within the same central pocket, where conserved lysine, arginine, and tyrosine residues recognize the shared phosphate group. A structural comparison with the outward-open conformation of the bacterial drug/metabolite transporter suggests a rocking-type motion of helix bundles, and molecular dynamics simulations support a model in which this helix rocking is tightly coupled to the substrate binding, to ensure strict $1: 1$ exchange. These results reveal the unique mechanism of sugar phosphate/phosphate exchange by TPT. 


\section{Introduction}

Photosynthetic organisms assimilate $\mathrm{CO}_{2}$ into organic compounds through the Calvin cycle ${ }^{1}$ and provide essential building blocks for all life on earth. In plants and algae, fixed carbons are exported from the chloroplast in the form of triose phosphate (triose- $\mathrm{P})^{2,3}$. This export is accomplished by the triose-phosphate/phosphate translocator (TPT) ${ }^{4-6}$, which catalyzes the exchange of triose-P, 3-phosphoglycerate (3-PGA) and inorganic phosphate (Pi) across the chloroplast inner envelope membrane (Extended Data Fig. 1a).

TPT belongs to the plastidic phosphate translocator (pPT) family ${ }^{7}$, whose members are widespread across all photosynthetic eukaryotes ${ }^{5}$, as well as in other organisms with plastids ${ }^{8}$. Land plants possess four pPT subtypes, including the phosphoenolpyruvate/phosphate translocator (PPT) ${ }^{9}$, the glucose-6-phosphate/phosphate translocator $(\mathrm{GPT})^{10}$ and the xylulose-5-phosphate/phosphate translocator $(\mathrm{XPT})^{11}$, which transport different sugar phosphates and function in various metabolic pathways (Extended Data Fig. 1b, c). These plant pPTs play crucial roles in crop metabolism ${ }^{12,13}$, and therefore they are regarded as attractive targets of genetic manipulation for improving crop productivity $^{14,15}$. The pPTs are also found in apicomplexan parasites ${ }^{16,17}$, which cause toxoplasmosis and malaria in humans. Since these apicomplexan pPTs are essential for the survival of the parasites $^{18,19}$, they are potential drug targets for parasitic infections ${ }^{20}$. All pPT proteins catalyze strict 1:1 exchange reactions ${ }^{3}$, and thereby guarantee the total phosphate balance of the plastid and the $-3-$ 
cytosol while allowing the transport of carbon and energy ${ }^{21}$.

TPT was discovered more than forty years $\mathrm{ago}^{3}$ and has been rigorously characterized at genetic and biochemical levels ${ }^{22}$. However, due to the lack of structural information, the mechanisms by which TPT recognizes the substrates and catalyzes the strict 1:1 exchange remain poorly understood.

\section{Results}

\section{Functional and structural analyses of TPT}

To elucidate the structure and the molecular mechanism of TPT, we systematically screened plant and algal pPTs for their expression and stability. Among the tested proteins, a pPT from the thermophilic red alga Galdieria sulphuraria (Gasu_21660; Extended Data Fig. 2a,b) showed excellent solution behavior. Its crystallization construct (residues 91-410) without the N-terminal chloroplast transit peptide showed the 'signature' $\mathrm{Pi} / \mathrm{Pi}$ homo-exchange activity ${ }^{23}$ in the liposome-based assay (Fig. 1a). We also confirmed the 3-PGA/Pi hetero-exchange activity (Fig. 1b). A competitive inhibition assay suggested that this pPT transports phosphorylated C3 compounds, but not phosphorylated C6 compounds (Fig. 1c). We further characterized its kinetic constants, and confirmed that its substrate specificity is comparable to that of higher plant TPTs: the Michaelis 
constant $\left(K_{\mathrm{m}}\right)$ for $\mathrm{Pi}$ is about $1.3 \mathrm{mM}$, and the inhibition constants $\left(K_{\mathrm{i}}\right)$ for triose-P, 3-PGA and phosphoenolpyruvate (PEP) are about 1.0, 0.6 and $8.3 \mathrm{mM}$, respectively (Fig. 1d,e). Based on these biochemical characterizations, we hereafter refer to this pPT as GsTPT2, although it was formerly named GsGPT ${ }^{23}$, based on the sequence similarity to GPT.

We crystallized recombinant GsTPT2 in the lipidic cubic phase ${ }^{24}$. Co-crystallization with high concentrations $(50-250 \mathrm{mM})$ of 3-PGA or Pi yielded diffraction-quality crystals belonging to the $P 2{ }_{1} 2_{1} 2$ space group, and we collected data from several hundred crystals, using a microfocus X-ray beam. A previous bioinformatics analysis suggested the classification of TPT into the drug/metabolite transporter (DMT) superfamily ${ }^{25}$, which contains various families of membrane proteins possessing 4, 5, 9 or 10 transmembrane (TM) helices. After extensive molecular replacement trials, we successfully obtained an initial solution with a poly-alanine model of the 10-TM DMT transporter $\mathrm{SnYddG}^{26}$. The final structures were determined at 2.1 and $2.2 \AA$ resolutions for the Pi- and 3-PGA-bound states, respectively (Extended Data Figs. 3,4). The protein regions of the two structures are almost identical, with an r.m.s.d. value of $0.18 \AA$ over $608 \mathrm{C} \alpha$ atoms.

The overall structure of GsTPT2 reveals a 10-TM helix topology with both the N-and C-termini on the stromal side (inside), rather than the previously predicted 6-9 TM helix topologies ${ }^{27}$ (Fig. 2a,b and Extended Data Fig. 5). GsTPT2 contains two 'inverted' structural repeats, comprising 
the N- and C-halves. Viewed from the intermembrane space side (outside), the five helices within the

$\mathrm{N}$ - and C- halves (i.e., TM1-5 and TM6-10) are arranged in counter-clockwise and clockwise manners, respectively (Extended Data Fig. 5). This fold is essentially similar to that of the bacterial DMT superfamily transporter $\mathrm{SnYddG}^{26}$, despite the low sequence identity (13.9\%), suggesting that this "10-TM DMT fold" could be conserved across all putative 10-TM members of the DMT superfamily $^{25}$ (Extended Data Fig. 6). In contrast to the 'outward-open' conformation of SnYddG, the current structure of GsTPT2 shows that its substrate-binding site is occluded from both sides of the membrane, revealing the 'occluded' conformation of a DMT protein for the first time.

Although the purified GsTPT2 protein is monomeric in solution, GsTPT2 forms a dimer in the crystallographic asymmetric unit (Extended Data Fig. 7). The inter-protomer interaction involves polar interactions at TM5, TM10 and a short $\beta$-strand connecting TM4 and TM5, and hydrophobic contacts through the lipid molecules bound at the interface. The same topological orientation of the monomers within the membrane suggests that this dimeric assembly could be physiologically relevant $^{28}$, although we cannot exclude the possibility that this is a crystallization artifact.

\section{3-PGA and Pi recognition}

The electron density maps clearly showed that 3-PGA and Pi are bound to the same site located halfway across the membrane, as if trapped in a central 'cage' formed by TM1-4 and TM6-9 
(Extended Data Fig. 8). The phosphate moiety of both ligands is identically recognized by ionic bonds with Lys204, Lys362 and Arg363 and a hydrogen bond with Tyr339 (Fig. 2c,d). While the three oxygen atoms of the phosphate (P-O2, O3 and $\mathrm{O} 4)$ are directly recognized by these sidechains, the remaining oxygen atom (P-O1) is not directly recognized. In the 3-PGA-bound structure, this P-O1 is attached to the glycerate group, which extends into the space on the opposite side of the phosphate moiety and forms specific interactions with protein sidechains (Fig. 2c). The carboxyl group on the $\mathrm{C} 1$ atom forms an ionic bond with the sidechain of His185, and the hydroxyl group on the $\mathrm{C} 2$ atom hydrogen bonds with the sidechain of Tyr339. In addition, the $\mathrm{C} 2$ and $\mathrm{C} 3$ atoms form hydrophobic contacts with the sidechains of Thr188, Phe192 and Phe263 (Extended Data Fig. 8).

In the Pi-bound structure, the corresponding space near P-O1 is occupied by three water molecules (Fig. 2d). These water molecules form polar interactions with His185 and Tyr339, contributing to the indirect recognition of P-O1. Notably, the positions of these water molecules roughly correspond to those of the three oxygen atoms of the glycerate moiety of 3-PGA, mimicking the organic carbon structure. This water-mediated hydrogen-bonding network is likely to lower the energy of the Pi-bound state and could explain why Pi, which lacks a sugar moiety, is transported with a similar affinity to those of other sugar phosphates ${ }^{21}$.

The observed binding mode of 3-PGA suggests that triose-P can be recognized in a similar manner. Indeed, modelling of triose-P into the crystal structure indicates a good fit, with the oxygen 
atoms at the $\mathrm{C} 1$ and $\mathrm{C} 2$ positions forming similar polar interactions with His 185 and Tyr339 (Fig. 2e).

Therefore, the structures suggest that triose-P, 3-PGA and Pi, the three major counter-substrates of TPT, are similarly recognized in a single pocket. The structures also reveal that this pocket could not accommodate two or more phosphate moieties at a time, explaining why pyrophosphate or bisphosphate compounds are not readily transported across the chloroplast envelope membrane ${ }^{3}$.

\section{Similarity and diversity of pPT subtypes}

The four phosphate-binding residues identified here are strictly conserved in all higher plant

pPTs (Extended Data Fig. 2a). To examine their functional importance, we performed mutational assays of these residues (Fig. 2f). All of the tested mutations exhibited greatly reduced $\mathrm{Pi} / \mathrm{Pi}$ homo-exchange activity, confirming their essential roles in phosphate transport. Besides these residues, a sequence comparison revealed that most of the residues near the phosphate moiety are also strictly conserved (Fig. 3a). In contrast, the residues distant from the phosphate, or near the sugar moiety, are varied among the different pPT subtypes (Fig. 3a). These findings suggest that the variant residues of the different $\mathrm{pPT}$ subtypes recognize the attached sugar moieties and thus determine their distinct substrate specificities, while the conserved residues similarly recognize the phosphate.

To better understand the substrate selectivities of the pPT family members ${ }^{5}$, we generated - 8- 
homology models of five representative pPTs, namely Arabidopsis thaliana TPT (AtTPT), PPT1

(AtPPT1), GPT1 (AtGPT1), and XPT (AtXPT) and Toxoplasma gondii APT (TgAPT). (Fig. 3 and

Extended Data Fig. 9). The AtTPT model suggests that the plant TPTs similarly recognize the substrates as in the current GsTPT2 structure, since the residues recognizing 3-PGA are highly conserved (His185, Lys204, Tyr339, Lys362 and Arg363 in AtTPT) (Fig. 3b,e). TPT prefers three-carbon compounds phosphorylated at the C3 position (triose-P and 3-PGA) to those phosphorylated at the $\mathrm{C} 2$ position (PEP and 2-PGA) by $\sim 10-$ fold $^{29}$. The AtTPT model indicates that the 'branched' C3 methylene group of PEP would sterically clash with the bulky Phe262 sidechain, explaining the lower preference for $\mathrm{PEP}^{9,30}$ (Fig. 3c). In contrast, the C3 carbon of PEP can be accommodated in the widened pocket of the AtPPT1 model, where Phe is replaced by Asn262, consistent with the PPT's preference for PEP ${ }^{9}$ (Fig. 3d). The apicomplexan pPTs, including TgAPT, PfipPT and PfopPT, have 'dual specificity', as they transport both triose-P and PEP with similar affinities $^{18,20}$. The TgAPT model explains its dual specificity well, as it can accommodate both triose-P and PEP (Extended Data Fig. 7c,d).

GPT transports glucose-6-phosphate (Glc-6-P), the largest substrate of all pPTs, as well as smaller substrates such as triose-P and 3-PGA ${ }^{10}$. The AtGPT1 model has the largest pocket space, which can accommodate the bulky C6 sugar (Fig. 3f), consistent with its broad substrate specificity. As compared with GPT, the AtXPT model has a rather small pocket, which might be suitable for the 
C5 sugar moiety of its substrate, xylulose-5-phosphate ${ }^{11}$ (Xul-5-P) (Fig. 3g). Collectively, our crystal structures and the homology models address how different pPT members transport distinct sugar phosphates and thereby play diverse roles in plastid metabolism ${ }^{5}$.

\section{Basis of strict 1:1 exchange}

Previous biochemical studies have shown that the transport by TPT is mediated by 'alternating-access ${ }^{29}$, in which the substrate-binding site is alternately exposed on either side of the membrane. In the current structure, the substrate is completely occluded from both sides of the membrane by the two gates (Fig. 4a). The 'outside gate' is formed by Phe192 and Ile197 on the tips of TM3 and TM4, and seals the substrate from the outside solvent (Fig. 4b). The 'inside gate' is formed by Leu347, Phe352 and Pro355 on the tips of TM8 and TM9, and similarly seals the substrate from the inside solvent (Fig. 4c). The helix ends of both gates are further capped by the conserved Lys128 and Lys271 residues (Fig. 4b,c).

To deduce the conformational change during the alternating-access, we compared this occluded structure with the available outward-open structure of the DMT transporter SnYddG. The structural superimposition revealed a prominent structural difference at TM3 and TM4 with a $\sim 30^{\circ}$ outward tilting in GsTPT2 (Fig. 4a), suggesting that these helices undergo rocking-type movements to open and close the outside gate. The pseudo-symmetric structure of GsTPT2 suggests that similar $-10-$ 
motion would occur in the symmetrical counterpart, TM8 and TM9, to open and close the inside gate (Supplementary Video 1).

To further understand the conformational changes, we performed molecular dynamics simulations of GsTPT2 in the presence or absence of the bound Pi (Fig. 5 and Extended Data Fig. 10). In the Pi-bound simulation, GsTPT2 did not undergo any significant structural change during $100 \mathrm{~ns}$ and remained in the occluded conformation (Fig. 5a,b). In contrast, in the apo simulation, GsTPT2 underwent rapid conformational changes within about $10 \mathrm{~ns}$ to the inward-open or outward-open conformations, and stably adopted these open conformations until the end of the simulation ( 100 ns) (Fig. 5c,d and Supplementary Video 2). These conformational changes strongly support our model proposed from the structural comparison with SnYddG, which involves the rocking-type movement of the helix bundles TM3-TM4-TM6 and TM1-TM8-TM9 to open and close the two gates.

The different behaviors in the Pi-bound and apo simulations suggest that the conformational change of GsTPT2 is completely dependent on the substrate binding (Fig. 6). Without a substrate, due to the electrostatic repulsion between the cationic residues (Lys204, Lys362 and Arg363) in the middle of the helix bundles, GsTPT2 prefers the outward- or inward-open states, as shown in the MD simulation. In contrast, phosphate or organic phosphate binding allows the close approximation of these cationic residues and thus leads to the occluded state, as in the current crystal structures. This 
ligand-dependent conformational change ensures the substrate-dependent transition between the inward- and outward-open states, and thus explains the strict 1:1 exchange kinetics of the pPTs ${ }^{21}$.

The proposed coupling mechanism between the substrate binding and the conformational change is quite different from the transport mechanism proposed for YddG, another 10-TM member of the DMT superfamily. YddG is a uniporter ${ }^{26}$ that permeates substrates down a concentration gradient, indicating the lack of structural coupling. This difference could be explained by the composition of its substrate-binding site. The substrate-binding pocket of YddG mostly consists of hydrophobic residues $^{26}$, which would lack electrostatic repulsion. YddG can thus adopt the occluded state without any substrate, consistent with its uniporter function. Therefore, even though GsTPT2 and YddG share the similar 10-TM DMT fold, the different compositions of their substrate-binding sites result in distinct transport mechanisms.

In conclusion, we determined the high-resolution structures of TPT in complex with two counter-substrates. The structures resolve the long-standing controversy over its helix topology ${ }^{4,27}$ and provide the framework to address its substrate recognition and strict 1:1 exchange mechanism. Further mechanistic understanding of the $\mathrm{pPT}$ family members could provide opportunities to engineer chloroplast transporters for improving crop productivity ${ }^{14,15}$, or to develop new drugs targeting plastid organelles of apicomplexan parasites ${ }^{20}$. 


\section{Author contributions}

Y.L. designed the research, expressed, purified and crystallized GsTPT2, determined the structures, and performed biochemical assays. Y.L., K.Y., and K.H. collected and processed diffraction data. T.N. and K.K. assisted with the structure determination. M.T. performed the molecular dynamics simulations. A.M. prepared G. sulphuraria cDNA. S.N. and K.T. performed the SEC-MALLS experiment. Y.L., T.N., R.I., and O.N. wrote the manuscript with help from all authors. O.N. directed and supervised all of the research.

\section{Acknowledgements}

We thank H. Nishimasu for comments on the manuscript; A. Kurabayashi, K. Ogomori, W.

Shihoya and R. Taniguchi for technical assistance; and the beam-line scientists at SPring-8 BL32XU for assistance in data collection. The diffraction experiments were performed at SPring-8 BL32XU (proposals 2015B0119 and 2015B2057). Computations of MD simulations were partially performed on the NIG supercomputer at ROIS National Institute of Genetics. This work was supported by grants from the Platform for Drug Discovery, Informatics and Structural Life Science by the Ministry of Education, Culture, Sports, Science and Technology (MEXT), JSPS KAKENHI (Grant Nos. 24227004, 25291011), the FIRST program, and a Grant-in-Aid for JSPS Fellows. 


\section{Figure legends}

\section{Figure 1 | Functional characterization of GsTPT2}

(a) $\mathrm{Pi} / \mathrm{Pi}$ homo-exchange activity of GsTPT2. Liposomes were reconstituted with yeast membranes expressing GsTPT2, and the time-dependent uptake of $\left[{ }^{32} \mathrm{P}\right]-\mathrm{Pi}$ was measured in the presence (filled circles) or absence (open squares) of internal Pi (30 mM). Error bars are s.e.m. $(n=3)$.

(b) Counter-flow assay. The uptake of $\left[{ }^{32} \mathrm{P}\right]-\mathrm{Pi}$ was measured in the presence of the indicated internal substrates $(30 \mathrm{mM})$. Control experiments were performed with membranes from yeast cells harboring empty vector. Error bars are s.e.m. $(n=3)$.

(c) Competitive inhibition assay. The uptake of $\left[{ }^{32} \mathrm{P}\right]-\mathrm{Pi}$ was assayed in the presence of the indicated competitive inhibitors $(40 \mathrm{mM})$ in the external solution. Error bars are s.e.m. $(\mathrm{n}=3)$.

(d) Concentration-dependent uptake of $\left[{ }^{32} \mathrm{P}\right]-\mathrm{Pi}$. Error bars are s.e.m. $(\mathrm{n}=3)$.

(e) Kinetic constants of GsTPT2 and the higher plant pPTs. The Michaelis constant $\left(K_{\mathrm{m}}\right)$ of GsTPT2 was calculated from the experiment shown in Figure 1d. Inhibitor constants $\left(K_{\mathrm{i}}\right)$ of GsTPT2 were evaluated at two different Pi concentrations with increasing inhibitor concentrations. Data are mean \pm s.e.m. $(n=3)$; n.d., not detectable. Values for the plant and apicomplexan pPTs were adopted from refs. ${ }^{9-11,18}$. 


\section{Figure 2 | Overall structure and substrate recognition of GsTPT2}

(a, b) Ribbon representations of the 3-PGA-bound (a) and the Pi-bound (b), occluded structures. IEM denotes the chloroplast inner envelope membrane.

(c, d) Close-up view of the 3-PGA-binding site (c) and the Pi-binding site (d). Dotted lines indicate polar interactions.

(e) Model of triose-P (dihydroxyacetone phosphate) binding.

(f) Liposome-based mutational analysis. The levels of $\left[{ }^{32} \mathrm{P}\right]-\mathrm{Pi}$ uptake by GsTPT2 mutants were compared to that of the wild-type. Error bars are s.e.m. $(n=3)$. Western blotting confirmed the comparable expression levels of the wild type and mutant proteins (small inset).

\section{Figure 3 | Comparison of the pPT subtypes}

(a) Amino-acid sequence conservation of the substrate-binding site of GsTPT2. Conserved residues are colored violet and non-conserved residues are cyan.

(b-g) Homology-modelled structures of the substrate-binding sites of the pPTs. Key residues involved in substrate recognition are shown as stick models. Substrate molecules were modelled manually, based on the coordination of 3-PGA in GsTPT2. Protein surfaces are shown for the regions 
around the substrate. In (c), the C3 carbon of PEP sterically clashed with the sidechain of Phe262 in the AtTPT model, indicating non-preferable binding.

\section{Figure 4 | Occluded structure and alternating-access}

(a) Superimposition of the occluded GsTPT2 structure and the outward-open SnYddG structure (PDB 5I20). The substrate and gate-forming residues are shown as CPK models. The red arrow highlights the putative rocking-type movements in TM3 and TM4.

(b) Close-up view of the outside gate.

(c) Close-up view of the inside gate.

\section{Figure 5 | Molecular dynamics simulations}

(a) R.m.s.d. plot of each monomer (Mol A and B) in the Pi-bound and apo simulations.

(b-d) Comparison of the starting structures (0 ns, transparent) and the final structures (100 ns, opaque) in the simulations. Red arrows highlight helix movements.

\section{Figure 6 | Proposed model for the strict 1:1 exchange}

(a-d) Illustration of a hypothetical conformational cycle of GsTPT2. Substrate binding $-16-$ 
enables the association of the helix bundles (a,c), triggering the conformational transition. Without a

substrate, the translocator cannot undergo the conformational transition due to the electrostatic repulsion $(b, d)$.

\section{References}

1 Bassham, J. A. Photosynthetic carbon metabolism. Proc. Natl. Acad. Sci. U. S. A. 68, 2877-2882 (1971).

2 Stocking, C. R. \& Larson, S. A chloroplast cytoplasmic shuttle and the reduction of extraplastid NAD. Biochem. Biophys. Res. Commun. 37, 278-282 (1969).

3 Heldt, H. W. \& Rapley, L. Specific transport of inorganic phosphate, 3-phosphoglycerate and dihydroxyacetonephosphate, and of dicarboxylates across the inner membrane of spinach chloroplasts. FEBS Lett. 10, 143-148 (1970).

4 Flügge, U. I. et al. The triose phosphate-3-phosphoglycerate-phosphate translocator from spinach chloroplasts: nucleotide sequence of a full-length cDNA clone and import of the in vitro synthesized precursor protein into chloroplasts. EMBO J. 8, 39-46 (1989).

5 Weber, A. P. \& Linka, N. Connecting the plastid: transporters of the plastid envelope and their role in linking plastidial with cytosolic metabolism. Annu. Rev. Plant Biol. 62, 53-77 (2011).

6 Flügge, U. I. et al. The major chloroplast envelope polypeptide is the phosphate translocator and not the protein import receptor. Nature 353, 364-367 (1991).

7 Knappe, S., Flügge, U. I. \& Fischer, K. Analysis of the plastidic phosphate translocator gene family in Arabidopsis and identification of new phosphate translocator-homologous transporters, classified by their putative substrate-binding site. Plant Physiol. 131, 1178-1190 (2003).

8 Weber, A. P., Linka, M. \& Bhattacharya, D. Single, ancient origin of a plastid metabolite translocator family in Plantae from an endomembrane-derived ancestor. Eukaryot. Cell 5, 609-612 (2006).

9 Fischer, K. et al. A new class of plastidic phosphate translocators: A putative link between primary and secondary metabolism by the phosphoenolpyruvate/phosphate antiporter. Plant Cell 9, 453-462 (1997).

10 Kammerer, B. et al. Molecular characterization of a carbon transporter in plastids from heterotrophic tissues: the glucose 6-phosphate/phosphate antiporter. Plant Cell 10, 105-117 
(1998).

11 Eicks, M., Maurino, V., Knappe, S., Flügge, U. I. \& Fischer, K. The plastidic pentose phosphate translocator represents a link between the cytosolic and the plastidic pentose phosphate pathways in plants. Plant Physiol. 128, 512-522 (2002).

12 Weber, A. P. \& Bräutigam, A. The role of membrane transport in metabolic engineering of plant primary metabolism. Curr. Opin. Biotechnol. 24, 256-262 (2013).

13 Wang, Y., Long, S. P. \& Zhu, X. G. Elements required for an efficient NADP-malic enzyme type C4 photosynthesis. Plant Physiol. 164, 2231-2246 (2014).

14 Zhang, L. et al. Overriding the co-limiting import of carbon and energy into tuber amyloplasts increases the starch content and yield of transgenic potato plants. Plant Biotechnol. J. 6, 453-464 (2008).

15 Cho, M. H., Jang, A., Bhoo, S. H., Jeon, J. S. \& Hahn, T. R. Manipulation of triose phosphate/phosphate translocator and cytosolic fructose-1,6-bisphosphatase, the key components in photosynthetic sucrose synthesis, enhances the source capacity of transgenic Arabidopsis plants. Photosynth. Res. 111, 261-268 (2012).

16 Mullin, K. A. et al. Membrane transporters in the relict plastid of malaria parasites. Proc. Natl. Acad. Sci. U. S. A. 103, 9572-9577 (2006).

17 Lim, L. \& McFadden, G. I. The evolution, metabolism and functions of the apicoplast. Philos. Trans. R. Soc. Lond. B. Biol. Sci. 365, 749-763 (2010).

18 Brooks, C. F. et al. The toxoplasma apicoplast phosphate translocator links cytosolic and apicoplast metabolism and is essential for parasite survival. Cell Host Microbe 7, 62-73 (2010).

19 Banerjee, T., Jaijyan, D. K., Surolia, N., Singh, A. P. \& Surolia, A. Apicoplast triose phosphate transporter (TPT) gene knockout is lethal for Plasmodium. Mol. Biochem. Parasitol. 186, 44-50 (2012).

20 Lim, L., Linka, M., Mullin, K. A., Weber, A. P. \& McFadden, G. I. The carbon and energy sources of the non-photosynthetic plastid in the malaria parasite. FEBS Lett. 584, 549-554 (2010).

21 Facchinelli, F. \& Weber, A. P. The metabolite transporters of the plastid envelope: an update. Front. Plant Sci. 2, 50 (2011).

22 Heldt, H. W. Three decades in transport business: studies of metabolite transport in chloroplasts - a personal perspective. Photosynth. Res. 73, 265-272 (2002).

23 Linka, M., Jamai, A. \& Weber, A. P. Functional characterization of the plastidic phosphate translocator gene family from the thermo-acidophilic red alga Galdieria sulphuraria reveals specific adaptations of primary carbon partitioning in green plants and red algae. Plant Physiol. 148, 1487-1496 (2008).

24 Landau, E. M. \& Rosenbusch, J. P. Lipidic cubic phases: a novel concept for the $-18-$ 
crystallization of membrane proteins. Proc. Natl. Acad. Sci. U. S. A. 93, 14532-14535 (1996).

25 Jack, D. L., Yang, N. M. \& Saier, M. H., Jr. The drug/metabolite transporter superfamily. Eur. J. Biochem. 268, 3620-3639 (2001).

26 Tsuchiya, H. et al. Structural basis for amino acid export by DMT superfamily transporter YddG. Nature 534, 417-420 (2016).

27 Weber, A. P., Schwacke, R. \& Flügge, U. I. Solute transporters of the plastid envelope membrane. Annu. Rev. Plant Biol. 56, 133-164 (2005).

28 Flügge, U. I. Hydrodynamic properties of the Triton X-100-solubilized chloroplast phosphate translocator. BBA-Biomembranes 815, 299-305 (1985).

29 Gross, A., Bruckner, G., Heldt, H. W. \& Flügge, U. I. Comparison of the kinetic properties, inhibition and labelling of the phosphate translocators from maize and spinach mesophyll chloroplasts. Planta 180, 262-271 (1990).

30 Nozawa, A. et al. A Cell-Free Translation and Proteoliposome Reconstitution System for Functional Analysis of Plant Solute Transporters. Plant Cell Physiol. 48, 1815-1820 (2007). 


\section{Methods}

\section{Cloning, expression and purification}

Total cDNA from G. sulphuraria was prepared from autotrophically grown cells. The region of GsTPT2 (GI: 194462447) encoding residues 91-410 was amplified from the cDNA and subcloned into a modified pFastbac vector, with a C-terminal TEV cleavage site, EGFP and a His ${ }_{10}$-tag. Recombinant baculoviruses were produced with the Bac-to-Bac system (Invitrogen), and were used to infect $\mathrm{Sf} 9$ cells at a density of $2-3 \times 10^{6}$ cells $\mathrm{ml}^{-1}$. After growth for $48 \mathrm{~h}$ at $27^{\circ} \mathrm{C}$, the cells were harvested and sonicated in lysis buffer $(50 \mathrm{mM}$ Tris- $\mathrm{HCl}(\mathrm{pH} 8.0), 150 \mathrm{mM} \mathrm{NaCl}$ and protease inhibitors). The cell debris was removed by low-speed centrifugation (10,000 g, $10 \mathrm{~min})$, and the membrane fraction was collected by ultracentrifugation $(138,000 \mathrm{~g}, 1 \mathrm{~h})$.

The membrane fraction was solubilized in solubilization buffer $(20 \mathrm{mM}$ Tris- $\mathrm{HCl}(\mathrm{pH} 8.0)$, $300 \mathrm{mM} \mathrm{NaCl}, 1 \%$ (w/v) lauryl maltoside neopentyl glycol (LMNG) and $1 \mathrm{mM} \beta$-mercaptoethanol $(\beta-\mathrm{ME}))$ for $3 \mathrm{~h}$ at $4^{\circ} \mathrm{C}$. The supernatant was isolated by ultracentrifugation $(138,000 \mathrm{~g}, 30 \mathrm{~min})$ and subjected to immobilized metal ion affinity chromatography (IMAC) with Ni-NTA resin (Qiagen). The resin was washed with IMAC buffer (20 mM Tris- $\mathrm{HCl}(\mathrm{pH} 8.0), 300 \mathrm{mM} \mathrm{NaCl}, 0.05 \%$ LMNG, $1 \mathrm{mM} \beta$-ME and $30 \mathrm{mM}$ imidazole), and the protein was eluted with IMAC buffer supplemented with $300 \mathrm{mM}$ imidazole. The eluate was treated with TEV protease and dialyzed overnight against 
dialysis buffer (20 mM Tris- $\mathrm{HCl}(\mathrm{pH} 8.0), 300 \mathrm{mM} \mathrm{NaCl}, 0.01 \%$ LMNG and $1 \mathrm{mM} \beta-\mathrm{ME}$ ). The cleaved EGFP-His ${ }_{10}$ and TEV protease were removed by reverse IMAC with Ni-NTA. The protein was concentrated to 2-3 mg ml${ }^{-1}$ using a $50 \mathrm{kDa}$ MWCO concentrator (Millipore), and further purified by size-exclusion chromatography (SEC) on a Superdex 200 Increase 10/300 column (GE Healthcare) in SEC buffer (10 mM Tris- $\mathrm{HCl}(\mathrm{pH} 8.0), 150 \mathrm{mM} \mathrm{NaCl}, 0.01 \%$ LMNG and $1 \mathrm{mM}$ $\beta-\mathrm{ME})$. The peak fractions were collected, concentrated to $10-20 \mathrm{mg} \mathrm{ml}^{-1}$, flash-frozen in liquid nitrogen and stored at $-80^{\circ} \mathrm{C}$ until crystallization.

\section{Size exclusion chromatography coupled to multi-angle laser light scattering (SEC-MALLS)}

The instrument setup for the SEC-MALLS experiment consisted of an Agilent 1100 Series

HPLC system connected in series with a Shimadzu SPD-10Avp UV absorbance detector, a Wyatt

DAWN HELEOS 8+ light scattering detector and a Shodex RI 101 refractive index detector.

Analytical size-exclusion chromatography was performed at $25^{\circ} \mathrm{C}$ on a Superdex $20010 / 300$ column equilibrated with buffer containing $10 \mathrm{mM}$ Tris- $\mathrm{HCl}(\mathrm{pH} 8.0), 150 \mathrm{mM} \mathrm{NaCl}$ and $0.01 \%$ LMNG. A $90 \mu \mathrm{l}$ portion of the purified GsTPT2 sample $\left(1.5 \mathrm{mg} \mathrm{ml}^{-1}\right)$ was injected into the column and eluted at $0.5 \mathrm{ml} \mathrm{min}{ }^{-1}$. Elution was monitored in line with the three detectors, which simultaneously measured UV absorption, light scattering and refractive index. A $658 \mathrm{~nm}$ laser was used in the light scattering $-21-$ 
measurement. Molecular masses were calculated using the three-detector method ${ }^{31}$, as implemented in the ASTRA software package (Wyatt Technology).

\section{Crystallization}

Purified samples were thawed and mixed with 1-oleoyl-R-glycerol (monoolein), at a protein to lipid ratio of 2:3 (w/w), to prepare the lipidic cubic phase (LCP), as previously described ${ }^{32}$.

Crystallization experiments were performed with 96-well glass sandwich plates (Molecular Dimensions), using a Gryphon LCP robot (Art Robbins Instruments). Typically, $50 \mathrm{nl}$ of protein-laden LCP drops were overlaid with $800 \mathrm{nl}$ of precipitant solution. After extensive co-crystallization screening, needle-shaped crystals appeared under conditions containing high concentrations of 3-PGA or Pi. Optimized crystals of the 3-PGA-bound state were obtained in 35-40\% PEG200, 100 mM Na-citrate (pH 6.0), 50-100 mM citrate 3K and 50-100 mM 3-PGA.2Na. Optimized crystals of the Pi-bound state were obtained in 43-48\% PEG200, 50-100 mM MES-NaOH (pH 6.0) and 200-250 mM ( $\left.\mathrm{NH}_{4}\right)_{2} \mathrm{HPO}_{4}$. Crystals were harvested and flash-cooled in liquid nitrogen for data collection. 


\section{Data collection and structure determination}

X-ray diffraction experiments were performed at the micro-focus beamline BL32XU at SPring-8. The locations of well-diffracting crystals were identified by raster scanning, and data were collected for a $5-30^{\circ}$ wedge from each crystal. All diffraction data were processed with $\mathrm{XDS}^{33}$, and merged with XSCALE based on the hierarchical clustering analysis with BLEND ${ }^{34}$ or with the cross-correlation method as implemented in the KAMO software (https://github.com/keitaroyam/yamtbx).

For the determination of the Pi-bound structure, we performed molecular replacement trials with Phaser ${ }^{35}$ using various truncated structures of DMT proteins as search models. Initial solutions were obtained with a full-length polyalanine model of the YddG monomer (PDB 5I20). After initial refinement in PHENIX ${ }^{36}$, the resulting map (Rfree value 53\%) showed poor or no electron density for substantial portions of the structure, particularly for TM5, TM10 and all loop regions. These invisible segments were deleted from the model and rebuilt by multiple trials of manual modelling of new polyalanine helices using $\mathrm{COOT}^{37}$ and refinement with phenix.refine, to find the correct helix assignment. After subsequent rounds of model building and refinement, we could build ten helix backbones and several sidechains into the visible electron density. However, at this point, further model building did not improve the $R_{\text {free }}$ value or the quality of the electron density. We then noticed that the model was of a 'swapped' form of the protein, where the N-terminal repeat (TM1-5) and the $-23-$ 
C- terminal repeat (TM6-10) were inversely assigned to each other. We corrected this swapping by renumbering the residues in COOT and proceeded with further model building. After building the protein regions (residues 100-404), strong electron densities were observed within the central cavities of the two monomers, which were unambiguously assigned as bound Pi molecules. During the later stages of refinement, electron densities for water and lipid molecules were also identified. The structure was iteratively rebuilt and refined with COOT and PHENIX to achieve good stereochemistry and $R_{\text {free }}$ values (Extended Data Table 1). The 3-PGA-bound structure was determined using the Pi-bound GsTPT2 dimer as the starting model, and iteratively rebuilt and refined with COOT and PHENIX.

\section{Preparation of reconstituted liposomes}

Yeast membranes expressing recombinant proteins were prepared as previously described ${ }^{23}$, with slight modifications. The region of GsTPT2 encoding residues $91-410$ was cloned into a modified pYES2 vector, with a C-terminal His 6 tag. For mutant assays, mutations were introduced by a PCR-based method. The plasmids were transformed into Saccharomyces cerevisiae cells (strain BY4742). Transformed cells were grown in CSM-URA medium containing $2 \%$ raffinose, and protein expression was induced with $2 \%$ galactose when the culture reached an $\mathrm{OD}_{600}=0.6$. After growth for $22 \mathrm{~h}$ at $30^{\circ} \mathrm{C}$, the cells were harvested and disrupted in lysis buffer (50 mM Tricine-KOH $-24-$ 
(pH 7.5), $0.1 \mathrm{mM}$ phenylmethylsulfonyl fluoride and 5\% glycerol), using acid-washed glass beads (200-400 $\mu \mathrm{m}$; Sigma). Glass beads and cell debris were removed by low-speed centrifugation $(4,000$ g, $2 \mathrm{~min})$, and the membrane fraction was collected by ultracentrifugation $(138,000 \mathrm{~g}, 1 \mathrm{~h})$. The membrane pellet was resuspended in $50 \mathrm{mM}$ Tricine- $\mathrm{KOH}$ ( $\mathrm{pH} 7.5$ ), flash-frozen in liquid nitrogen and stored at $-80^{\circ} \mathrm{C}$ until use. Aliquots of the resuspended membranes were subjected to SDS-PAGE, and the His ${ }_{6}$-tagged recombinant proteins were detected by a western-blot analysis, using an anti-His-tag polyclonal antibody (code PM032; MBL).

Soybean L- $\alpha$-phosphatidylcholine (Avanti) in chloroform was dried into a thin film under a stream of nitrogen gas, and further dried under vacuum. Dried lipids were resuspended at $20 \mathrm{mg} \mathrm{ml}^{-1}$ in intra-liposomal solution (120 mM Tricine- $\mathrm{KOH}(\mathrm{pH} 7.5)$ and $\left.30 \mathrm{mM} \mathrm{NaH} \mathrm{PO}_{4}\right)$ or Pi-free intra-liposomal solution $(150 \mathrm{mM}$ Tricine- $\mathrm{KOH}(\mathrm{pH} 7.5))$, and sonicated for $5 \mathrm{~min}$ at $4^{\circ} \mathrm{C}$ to form unilamellar vesicles. This unilamellar vesicle solution was reconstituted with the yeast membranes at 19:1 (v/v), by the freeze-thaw procedure. The reconstituted liposomes were sonicated again for $5 \mathrm{~min}$ at $4^{\circ} \mathrm{C}$, to form unilamellar vesicles. The extra-liposomal solution was exchanged by gel-filtration on Sephadex G-50 (GE Healthcare) pre-equilibrated with $150 \mathrm{mM}$ Tricine-KOH (pH 7.5).

\section{Liposome assays}

The liposome assays were performed as previously described ${ }^{23}$, with slight modifications. 
For the time-dependent uptake assay, the reaction was started by mixing the reconstituted liposome solution with an equal volume of extra-liposomal solution (150 mM Tricine-KOH (pH 7.5) and $\left.1 \mathrm{mM}\left[{ }^{32} \mathrm{P}\right]-\mathrm{NaH}_{2} \mathrm{PO}_{4}\left(0.1 \mathrm{mCi} \mathrm{ml}^{-1}\right)\right)$. At different time points, liposomes were isolated by anion exchange on AG-1 X8 resin (acetate form, 200-400 dry mesh size; Bio-Rad), pre-equilibrated with $150 \mathrm{mM}$ sodium acetate. The radioactivity of the incorporated $\left[{ }^{32} \mathrm{P}\right]-\mathrm{Pi}$ was quantified by liquid scintillation counting. Mutant assays were performed with a similar procedure, and the total amounts of incorporated $\left[{ }^{32} \mathrm{P}\right]-\mathrm{Pi}$ were compared at $30 \mathrm{~min}$.

For the counter-flow assay, the liposomes containing $30 \mathrm{mM} \mathrm{Pi}, 30 \mathrm{mM}$ 3-PGA or no substrate were mixed with extra-liposomal solution containing $0.25 \mathrm{mM}\left[{ }^{32} \mathrm{P}\right]-\mathrm{Pi}$. The total amounts of incorporated $\left[{ }^{32} \mathrm{P}\right]-\mathrm{Pi}$ were compared at $3 \mathrm{~min}$.

For the competitive inhibition assay, the liposomes containing $30 \mathrm{mM}$ Pi were mixed with extra-liposomal solution containing $0.25 \mathrm{mM}\left[{ }^{32} \mathrm{P}\right]-\mathrm{Pi}$ and $40 \mathrm{mM}$ of the indicated competitive inhibitor. The total amounts of incorporated $\left[{ }^{32} \mathrm{P}\right]-\mathrm{Pi}$ were compared at $3 \mathrm{~min}$.

For the determination of kinetic constants, the Michaelis constant $\left(K_{\mathrm{m}}\right)$ for Pi was analyzed using various external concentrations of $\left[{ }^{32} \mathrm{P}\right]-\mathrm{Pi}(0.315-10 \mathrm{mM})$ and a fixed internal concentration of Pi (30 mM). Inhibitor constants $\left(K_{\mathrm{i}}\right)$ were assessed with two different external concentrations of $\left[{ }^{32} \mathrm{P}\right]-\mathrm{Pi}(0.5-2.5 \mathrm{mM})$ and four different concentrations of the indicated inhibitors $(0-10 \mathrm{mM})$. To assess the background uptake, control experiments were performed with membranes from yeast cells 
transformed with empty vector. Enzyme kinetic data were analyzed by non-linear regression fitting, as implemented in the GraphPad Prism 7 software.

\section{Molecular dynamics simulation}

The simulation system included the GsTPT2 dimer, 1-phosphoryl-2-oleoylphosphatidylcholine (POPC), TIP3P water and $150 \mathrm{mM} \mathrm{NaCl}$. The disordered sidechains in the GsTPT2 crystal structure were modelled by COOT $^{37}$. To embed the protein within the POPC bilayer, the protocol described by Javanainen ${ }^{38}$ was used. One POPC molecule was placed in the GsGPT dimerization interface, corresponding to the two monoolein molecules in the crystal structure. Finally, the periodic boundary systems, including 136,668 (with Pi) and 136,652 (without Pi) atoms, with the size of $90.7 \times 147.9 \times 100.0 \AA$, were prepared. The net charge of the solute was neutralized with sodium and chloride ions. The molecular topologies and force field parameters from CHARMM36 (ref. ${ }^{39}$ ) were used. Molecular dynamics simulations were performed by the program Gromacs, version 5.0.5 (ref. ${ }^{40}$ ). First, energy minimization was performed using the steepest descent, with a cut-off of $1,000.0 \mathrm{~kJ} \mathrm{~mol}^{-1} \mathrm{~nm}^{-1}$. Next, random velocities were assigned according to a Maxwell distribution, at a temperature of $310 \mathrm{~K}$ for each atom, and an equilibration run (eq1) was performed for $100 \mathrm{ps}$ in the canonical $(N V T)$ ensemble $(310 \mathrm{~K}, 90.7 \times 147.9 \times 100.0 \AA$ volume $)$. Finally, an equilibration run (eq2) was performed for $1,000 \mathrm{ps}$ in the isothermal-isobaric (NPT) ensemble $-27-$ 
( $310 \mathrm{~K}, 1 \mathrm{bar})$. The positions of non-hydrogen atoms in the protein and phosphates were restrained with a force constant of $1,000 \mathrm{~kJ} \mathrm{~mol}^{-1} \mathrm{~nm}^{-2}$, in the minimization and equilibration runs. Production runs were performed for $100 \mathrm{~ns}$ in the NPT ensemble (310 K, 1 bar). The same simulation was performed twice with different initial velocities, and similar results were obtained. Constant temperature was maintained by using $\mathrm{V}_{\text {-rescaling }}{ }^{41}$ with a time constant of 0.1 ps in eq1, and a Nosé-Hoover thermostat ${ }^{42,43}$ with a time constant of 0.5 ps in eq 2 and the production runs. Pressure was controlled with semiisotropic coupling to a Parrinello-Rahman barostat ${ }^{44}$, with a time constant of 5.0 ps and a compressibility of $4.5 \times 10^{-5}$ bar $^{-1}$. The LINCS algorithm ${ }^{45}$ was used for bond constraints. Long range electrostatic interactions were calculated with the particle mesh Ewald method ${ }^{46}$. 


\section{Extended Data}

\section{Extended Data Figure 1 | Roles of the pPTs in plastid metabolism}

(a) The function of TPT in chloroplasts. Under physiological conditions, TPT catalyzes either triose-P/Pi or triose-P/3-PGA exchange across the chloroplast inner envelope membrane. The former reaction delivers a carbon skeleton to the cytoplasm and transports Pi back into the chloroplast for ATP regeneration. The latter reaction, known as the triose-P/3-PGA shuttle ${ }^{2}$, indirectly exports chemical energy (ATP and NADPH) without the net transport of carbon. OEM and IEM denote the outer and inner envelope membranes, respectively.

(b) The functions of other pPT subtypes. PPT exchanges PEP with Pi and plays important roles in amino acid and fatty acid syntheses ${ }^{9}$. PPT is also a part of the $\mathrm{CO}_{2}$ concentration mechanism of $\mathrm{C} 4$ and CAM photosynthesis ${ }^{47}$. GPT and XPT exchange Glc-6-P and Xul-5-P with Pi, respectively, and they play important roles in the starch biosynthesis and pentose-phosphate pathways ${ }^{10,11}$.

(c) Chemical structures of the pPT substrates.

\section{Extended Data Figure 2 | Sequence alignment of the pPTs}

(a) Amino acid sequence alignment of the pPTs.

(b) Sequence identity matrix of the pPTs. Identities were calculated for the mature - 29- 
translocator regions (corresponding to residues 101-410 in GsTPT2).

\section{Extended Data Figure 3 | Crystallization, data collection and structure determination of GsTPT2}

(a) Co-crystals of GsTPT2 and 3-PGA.

(b) Diffraction image of the 3-PGA-bound state.

(c) Magnified image of the region enclosed by the square in (b).

(d) Co-crystals of GsTPT2 and Pi.

(e) Diffraction image of the Pi-bound state.

(f) Magnified image of the region enclosed by the square in (e).

(g) Crystal packing of the 3-PGA-bound structure.

(h) Crystal packing of the Pi-bound structure.

(i) Fo-Fc omit map of 3-PGA, contoured at $4.0 \sigma$.

(j) $F_{\mathrm{O}-\mathrm{Fc}}$ omit map of $\mathrm{Pi}$, contoured at $4.0 \sigma$.

(k) Superimposition of the monomers in the 3-PGA-bound structure.

(1) Superimposition of the monomers in the Pi-bound structure

(m) Superimposition of the Pi-bound and 3-PGA-bound dimers. 


\section{Extended Data Figure 4 | Electron density map}

(a) $2 F_{\mathrm{O}}-F_{\mathrm{c}}$ map (contoured at $1.7 \sigma$ ) of the 3-PGA-bound structure. TM1, 8, and 9 are not shown for clarity.

(b) $2 F_{\mathrm{o}}-F_{\mathrm{c}}$ map (contoured at $1.7 \sigma$ ) of the Pi-bound structure.

\section{Extended Data Figure 5 | Structural pseudo-symmetry of GsTPT2}

(a) Transmembrane topology of GsTPT2. TM1 to TM10 are color-coded in rainbow. The asterisk highlights the position of the substrate-binding site.

(b) Superimposition of the two structural repeats. The N-terminal repeat (residues 100-245) and the C-terminal repeat (residues 246-404) were superimposed by the SSM algorithm ${ }^{48}$. TM5 and TM10 did not superimpose well, indicating local asymmetry.

\section{Extended Data Figure 6 | Structural similarity of the two DMT superfamily transporters}

(a) Sequence comparison between GsTPT2 and SnYddG. The two sequences were aligned by using Clustal omega ${ }^{49}$ and manually adjusted based on the secondary structure.

(b, c) Stereo views of the superimposition between GsTPT2 and SnYddG. The outer halves $-31-$ 
of TM3, TM4 and TM6 show structural differences relevant to the proposed gating mechanism. TM5

and TM10 also show large shifts, which are not relevant to the gating mechanism. The structures were superimposed by the SSM algorithm ${ }^{48}$. TM1-4 and TM6-9 were used for secondary structure element calculations, and $168 \mathrm{C} \alpha$ atoms were used for the final three-dimensional alignment.

\section{Extended Data Figure 7 | Dimerization of GsTPT2 in the crystal}

(a) $2 F_{\mathrm{O}}-\mathrm{Fc}_{\mathrm{c}}$ map (contoured at $1.0 \sigma$ ) of the six monoolein molecules identified near the dimer interface.

(b) Lipid-protein interactions. Four monoolein molecules mediate the hydrophobic contacts between the two protomers.

(c, d) Protein-protein interactions. Polar sidechains form hydrogen bonds between the protomers (c). The loops connecting TM4 and TM5 form a short, two-stranded $\beta$-sheet between the two protomers (d). Dotted lines indicate polar interactions.

(e) SEC-MALLS analysis of GsTPT2. The three chromatograms show the readings of the UV absorption, refractive index and light scattering detectors. The traces were normalized to the peak maxima. The cyan and red curves in the light scattering chromatogram indicate the calculated molecular masses of the protein-detergent complex and the protein, respectively (Mc and Mp; values are given on the right axis). The black arrow highlights the position of the elution peak of GsTPT2. 
The refractive index increments $(\mathrm{dn} / \mathrm{dc})$ of the protein and the detergent were assumed to be 0.185 and 0.132 , respectively ${ }^{50}$.

(f) Molecular mass values determined by the SEC-MALLS experiment or calculated from the amino acid sequence. The experimental protein mass was determined to be about $29.6 \mathrm{kDa}$, corresponding to the theoretical mass of the GsTPT2 monomer, $36.4 \mathrm{kDa}$.

\section{Extended Data Figure 8 | Substrate recognition}

(a) Stereo view of the 3-PGA binding site. Dotted lines indicate polar interactions. Red balls represent water molecules.

(b) Stereo view of the Pi binding site.

(c) Schematic diagram of the 3-PGA coordination.

\section{Extended Data Figure 9 | Structural basis of substrate specificity in the pPT subtypes}

(a-g) Homology-modelled structures of the substrate-binding sites of the pPTs. In (b-g), the substrate molecules were modelled manually, based on the coordination of 3-PGA in GsTPT2 (a).

Key residues involved in substrate recognition are shown as stick models. Protein surfaces are shown 
for selected regions around the substrates. In (b), the C3 carbon of the PEP model sterically clashed with the Phe263 sidechain in GsTPT2.

\section{Extended Data Figure 10 | Molecular dynamics simulations}

(a-d) Cut-away surface representations of the final structures (100 ns) in the simulations. We performed the apo and Pi-bound simulations independently twice. In the Pi-bound simulations, the translocator stably adopted the occluded conformation (a,b). In contrast, in the apo simulations, the two protomers adopted the inward- and outward-open conformations (c), or both adopted the inward-open conformation (d), suggesting that the presence or absence of the substrate in the central pocket significantly affected the translocator conformation, and supporting the substrate-dependent conformational change (discussed in the main text). In addition, the weak structural coupling of the two protomers ('inward-outward' or 'inward-inward') suggested that each protomer acts as an independent functional unit, consistent with the ping-pong type kinetics ${ }^{51}$.

(e-1), Comparison of the starting structures (0 ns, transparent) and the final structures (100 ns, opaque). 


\section{Extended Data Table 1}

Data collection and refinement statistics

\begin{tabular}{|c|c|c|}
\hline & 3-PGA-bound ${ }^{\text {a }}$ & Pi-bound $^{b}$ \\
\hline \multicolumn{3}{|l|}{ Data collection } \\
\hline Space group & $P 2{ }_{1} 2_{1} 2$ & $P 2{ }_{1}{ }_{1} 2$ \\
\hline \multicolumn{3}{|l|}{ Cell dimensions } \\
\hline$a, b, c(\AA)$ & $107.05,165.33,41.14$ & $106.55,164,97,41.42$ \\
\hline$\alpha, \beta, \gamma\left(^{\circ}\right)$ & $90,90,90$ & $90,90,90$ \\
\hline Resolution $(\AA)$ & $50.0-2.20(2.28-2.20)^{\mathrm{c}}$ & $50.0-2.10(2.18-2.10)$ \\
\hline $\mathrm{CC}_{1 / 2}$ & $0.994(0.698)$ & $0.995(0.653)$ \\
\hline$I / \sigma I$ & $13.3(1.3)$ & $11.3(1.1)$ \\
\hline Completeness (\%) & $100.0(99.9)$ & $99.8(100.0)$ \\
\hline Redundancy & $39.2(27.0)$ & $90.3(87.0)$ \\
\hline \multicolumn{3}{|l|}{ Refinement } \\
\hline Resolution $(\AA)$ & $50.0-2.20$ & $50.0-2.10$ \\
\hline No. reflections & 38,123 & 43,639 \\
\hline$R_{\text {work }} / R_{\text {free }}$ & $19.0 / 22.9$ & $19.5 / 22.6$ \\
\hline \multicolumn{3}{|l|}{ No. atoms } \\
\hline Protein & 4,713 & 4,721 \\
\hline Substrate & 22 & 10 \\
\hline Lipid & 426 & 558 \\
\hline Other & 235 & 283 \\
\hline \multicolumn{3}{|l|}{$B$-factors } \\
\hline Protein & 28.70 & 24.82 \\
\hline Substrate & 23.45 & 20.09 \\
\hline Lipid & 41.58 & 43.90 \\
\hline Other & 36.85 & 35.94 \\
\hline \multicolumn{3}{|l|}{ Ramachandran plot } \\
\hline Favored (\%) & 99.3 & 99.5 \\
\hline Allowed (\%) & 0.7 & 0.5 \\
\hline Outliers (\%) & 0 & 0 \\
\hline
\end{tabular}


${ }^{\mathrm{b}}$ Diffraction data were collected from 319 crystals.

${ }^{c}$ Values in parentheses are for highest-resolution shell.

\title{
Supplementary Videos
}

\section{Supplementary Video 1 | Conformational change of GsTPT2}

\section{Supplementary Video 2 | Molecular dynamics simulation of GsTPT2}

\author{
without Pi
}




\section{Additional references}

31 Hayashi, Y., Matsui, H. \& Takagi, T. Membrane protein molecular weight determined by low-angle laser light-scattering photometry coupled with high-performance gel chromatography. Methods Enzymol. 172, 514-528 (1989).

32 Caffrey, M. Crystallizing membrane proteins for structure determination: use of lipidic mesophases. Annu. Rev. Biophys. 38, 29-51 (2009).

33 Kabsch, W. XDS. Acta Crystallogr. D 66, 125-132 (2010).

34 Foadi, J. et al. Clustering procedures for the optimal selection of data sets from multiple crystals in macromolecular crystallography. Acta Crystallogr. D 69, 1617-1632 (2013).

35 McCoy, A. J. et al. Phaser crystallographic software. J. Appl. Crystallogr. 40, 658-674 (2007).

36 Adams, P. D. et al. PHENIX: a comprehensive Python-based system for macromolecular structure solution. Acta Crystallogr. D 66, 213-221 (2010).

37 Emsley, P., Lohkamp, B., Scott, W. G. \& Cowtan, K. Features and development of Coot. Acta Crystallogr. D 66, 486-501 (2010).

38 Javanainen, M. Universal Method for Embedding Proteins into Complex Lipid Bilayers for Molecular Dynamics Simulations. J. Chem. Theory Comput. 10, 2577-2582 (2014).

39 Klauda, J. B. et al. Update of the CHARMM all-atom additive force field for lipids: validation on six lipid types. J. Phys. Chem. B 114, 7830-7843 (2010).

40 Hess, B., Kutzner, C., van der Spoel, D. \& Lindahl, E. GROMACS 4: Algorithms for Highly Efficient, Load-Balanced, and Scalable Molecular Simulation. J. Chem. Theory Comput. 4, 435-447 (2008).

41 Bussi, G., Donadio, D. \& Parrinello, M. Canonical sampling through velocity rescaling. $J$. Chem. Phys. 126, 014101 (2007).

42 Nosé, S. A unified formulation of the constant temperature molecular dynamics methods. $J$. Chem. Phys. 81, 511 (1984).

43 Hoover, W. G. Canonical dynamics: Equilibrium phase-space distributions. Phys. Rev. A 31, 1695-1697 (1985).

44 Parrinello, M. \& Rahman, A. Polymorphic Transitions in Single-Crystals - a New Molecular-Dynamics Method. J. Appl. Phys. 52, $7182-7190$ (1981).

45 Hess, B., Bekker, H., Berendsen, H. J. C. \& Fraaije, J. G. E. M. LINCS: A linear constraint solver for molecular simulations. J. Comput. Chem. 18, 1463-1472 (1997).

46 Darden, T., York, D. \& Pedersen, L. Particle Mesh Ewald - an N.Log(N) Method for Ewald Sums in Large Systems. J. Chem. Phys. 98, 10089-10092 (1993).

47 Flügge, U. I., Hausler, R. E., Ludewig, F. \& Gierth, M. The role of transporters in supplying energy to plant plastids. J. Exp. Bot. 62, 2381-2392 (2011).

$$
\text { - } 37-
$$


48 Krissinel, E. \& Henrick, K. Secondary-structure matching (SSM), a new tool for fast protein structure alignment in three dimensions. Acta Crystallogr. Section D, Biol. Crystallogr. 60, 2256-2268 (2004).

49 Sievers, F. et al. Fast, scalable generation of high-quality protein multiple sequence alignments using Clustal Omega. Mol. Syst. Biol. 7, 539 (2011).

50 Rollauer, S. E. et al. Structure of the TatC core of the twin-arginine protein transport system. Nature 492, 210-214 (2012).

51 Flügge, U. I. Reaction mechanism and asymmetric orientation of the reconstituted chloroplast phosphate translocator. Biochim. Biophys. Acta 1110, 112-118 (1992). 
bioRxiv greprint doi: https://doi.org/10.1101/169169; this yersion posted August 18, 2017. The cepyright holder for this preprint (which was not certified by peer review) is the author/funder. All rights reserved. No reuse allowed without permission.
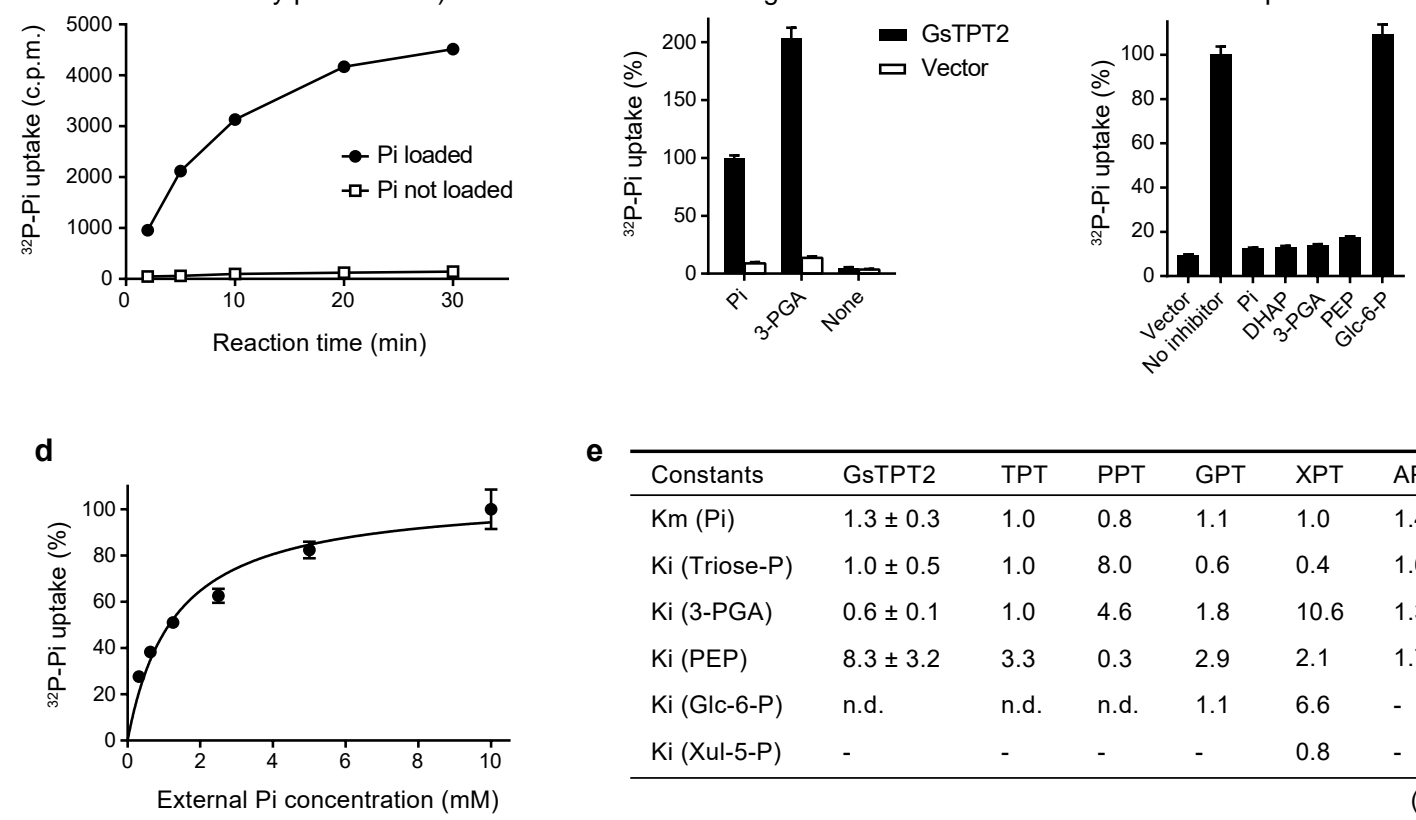

\begin{tabular}{lllllll}
\hline Constants & GsTPT2 & TPT & PPT & GPT & XPT & APT \\
\hline Km (Pi) & $1.3 \pm 0.3$ & 1.0 & 0.8 & 1.1 & 1.0 & 1.4 \\
Ki (Triose-P) & $1.0 \pm 0.5$ & 1.0 & 8.0 & 0.6 & 0.4 & 1.6 \\
Ki (3-PGA) & $0.6 \pm 0.1$ & 1.0 & 4.6 & 1.8 & 10.6 & 1.3 \\
Ki (PEP) & $8.3 \pm 3.2$ & 3.3 & 0.3 & 2.9 & 2.1 & 1.7 \\
Ki (Glc-6-P) & n.d. & n.d. & n.d. & 1.1 & 6.6 & - \\
Ki (Xul-5-P) & - & - & - & - & 0.8 & - \\
\hline
\end{tabular}


bippRiv preprint doi: https://doi.org/10.1101/169169; this version posted August 18, 2017. The copyright holder for this preprint (which was not
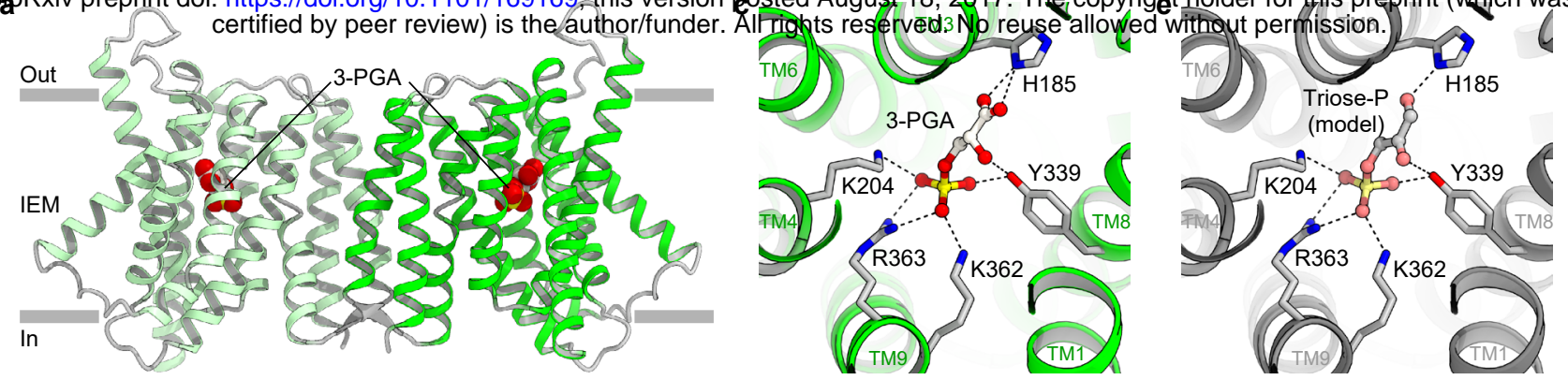

b
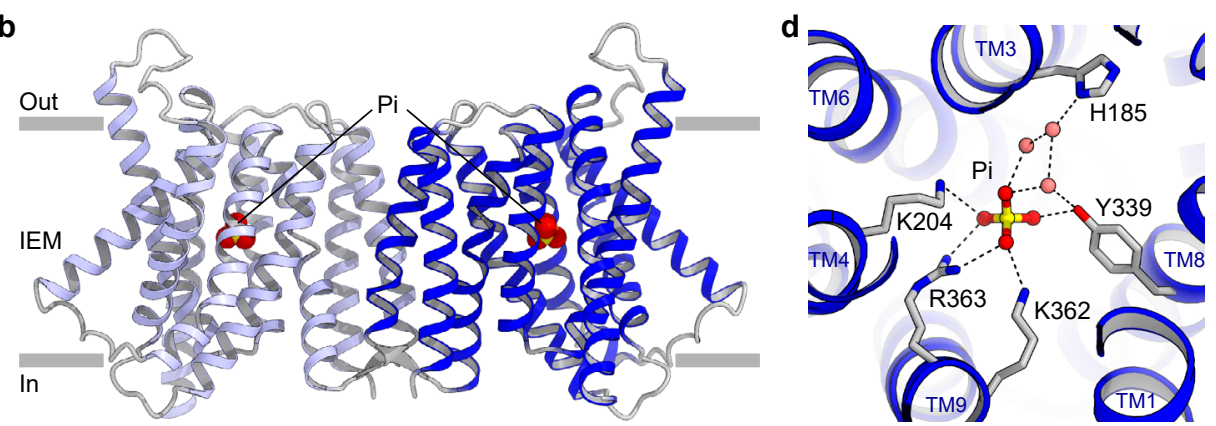

f

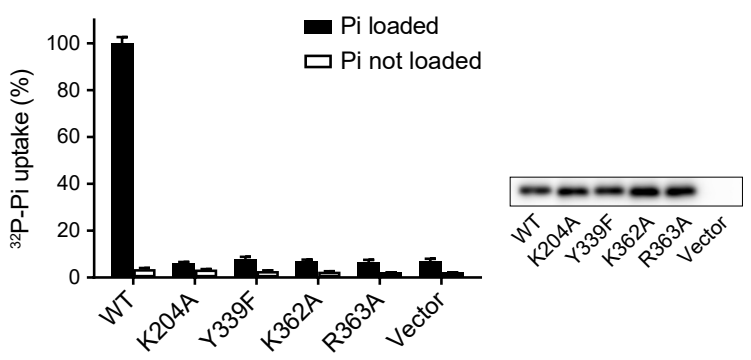


bioRxiv preprint doi: https://doi.org/10.1101/169169; this version posted August 18, 2017. The copyright holder for this preprint (which was not certified by peer review) is the author/funder. All rights reserved. No reuse allowed without permission.
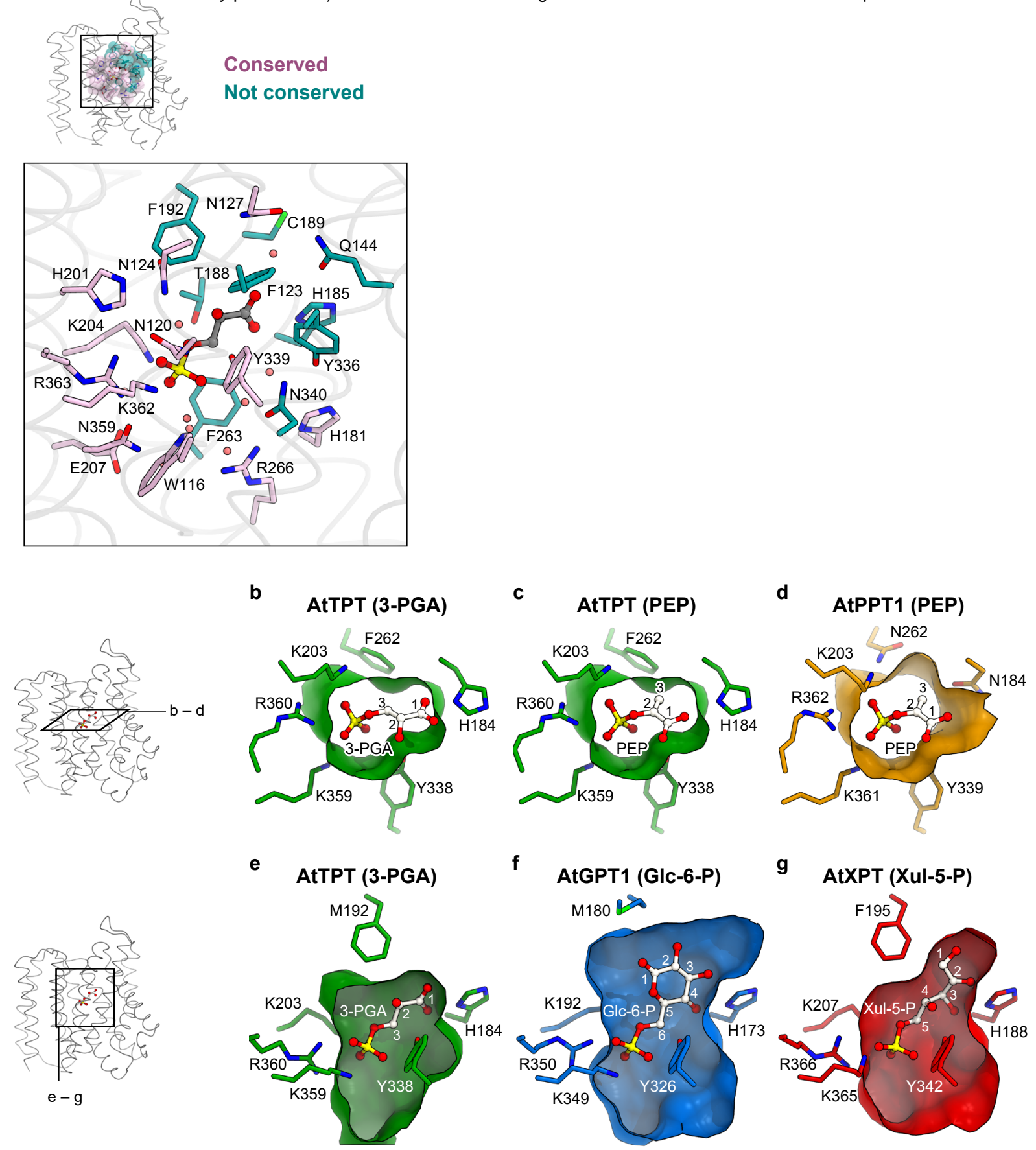
bioRxiv preprint doi: https://doi.org/10.1101/169169; this version posted August 18, 2017. The copyright holder for this preprint (which was not certified by peer review) is the author/funder. All rights reserved. No reuse allowed without permission.
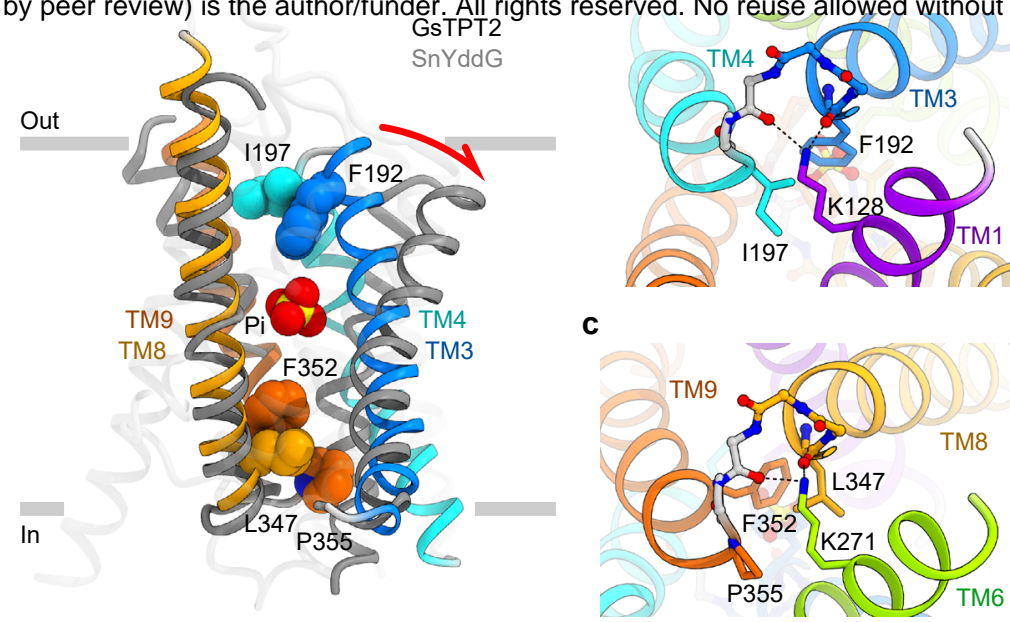

c

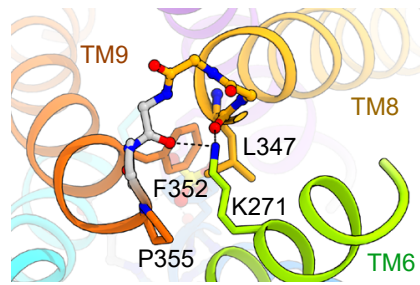


bioRxiv preprint doi: https://doi.org/101101/169169; this version posted August 18, 2017. The copyright holder for this preprint (which was not certified by peer reviews)_is the author/funder. All rights reserved. No reuse allowed without permission.

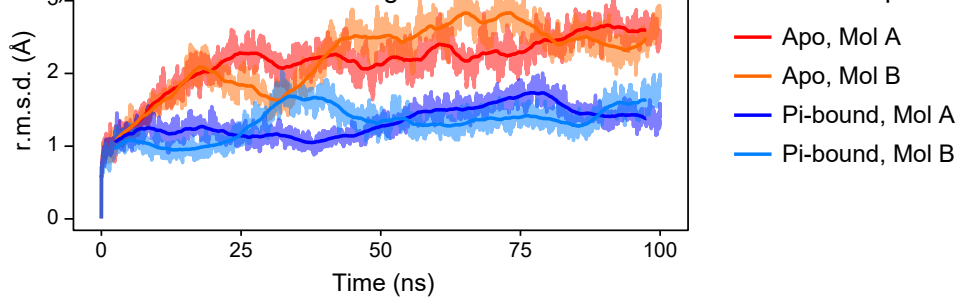

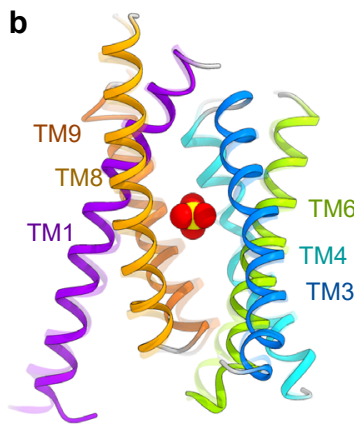

Occluded

(Pi-bound simulation, Mol A)
C

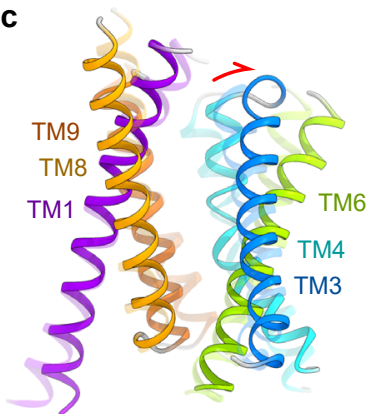

Outward-open

(Apo simulation, Mol A)

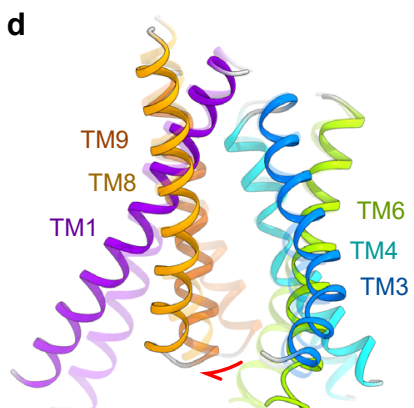

Inward-open

(Apo simulation, Mol B) 
bioRxiv preprint doi: https://doi.org/10.1101/169169; this version posted August 18, 2017. The copyright holder for this preprint (which was not certified by peer review) is the author/funder. All rights resefged. No reuse allowed without permission.

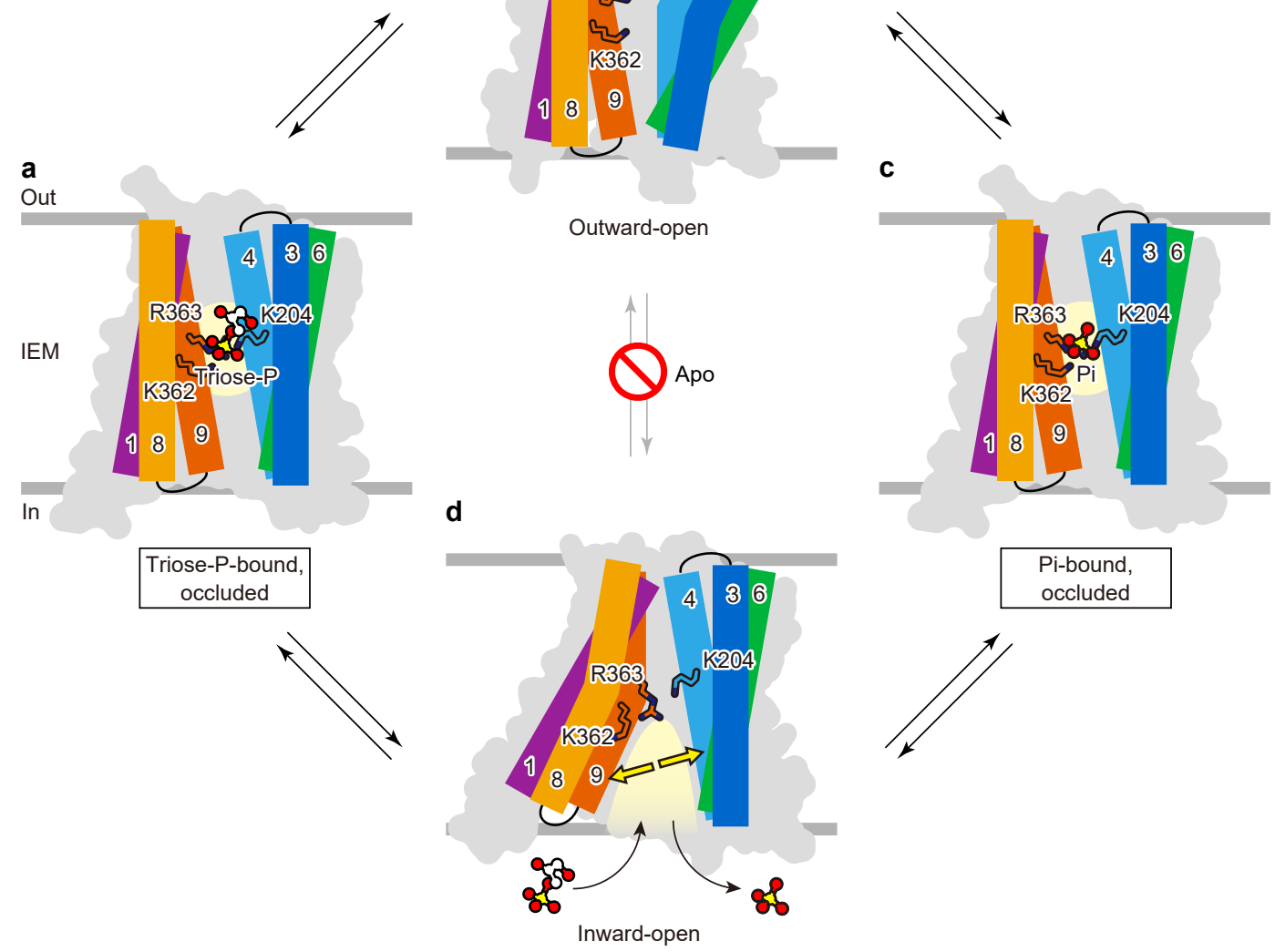

